\title{
Prevalence of Cigarette Smoking and Associated Factors among Male Citizens in Tehran, Iran
}

\author{
Aziz Kassani ${ }^{1}$, Abdolvahab Baghbanian ${ }^{2}$, Rostam Menati ${ }^{1}$, Jafar Hassanzadeh ${ }^{3}$, \\ Mohsen Asadi-Lari ${ }^{4}$, Walieh Menati ${ }^{1 *}$
}

\begin{abstract}
Background: Cigarette smoking is as the leading cause of cancer mortality and other chronic diseases in males worldwide. The prevalence of cigarette smoking is different across and within countries by age, education level, occupation, and so on. This study aimed to determine the prevalence of cigarette smoking and its relationship with individuals' demographic factors and BMI in adolescent men living in Tehran, Iran. Materials and Methods: This study involved secondary analysis of the 'Urban Health Equity Assessment and Response Tool-2' survey conducted in Tehran, Iran, among men aged 20+, 2011-2012. Using a multistage sampling method, 45,990 men were included in the study. The cigarette smoking status, BMI and demographic factors measured through a self-administered questionnaire. Chi-square, t-test, and logistic regression model were used to examine the relationships between the independents variables and cigarette smoking behavior, using SPSS software version 21. Results: In the total of 45,990 men, the overall prevalence of cigarette smoking was $14.6 \%$ (CI 95\%: 14.2914.94). Age (OR=0.96; CI 95\%:0.94-0.98), house ownership (OR=0.68; CI 95\%: 0.64-0.72), job status (OR=0.60; CI 95\%: 0.46-0.86), marital status (OR=0.42; CI 95\%: 0.39-0.47) and educational levels (OR=0.50; CI95\%: 0.45-0.54) were associated with the prevalence of cigarette smoking. However, associations with BMI, family size, residency years, and district were not statistically significant. Conclusions: Given the relatively high prevalence of cigarette smoking in the study population, policy interventions are required to address this major public health issue, with a focus on the population demographic influences.
\end{abstract}

Keywords: Cigarette smoking - body mass index- demographic factors - males - Tehan - Iran

Asian Pac J Cancer Prev, 17 (3), 1473-1478

\section{Introduction}

Before the beginning of widespread tobacco production and use in the late 19th century, the prevalence of noncommunicable diseases (NCDs) such as cardiovascular diseases, cancers, chronic respiratory diseases and diabetes was low. But they are now disproportionately affect low- and middle-income countries, where almost threequarters of NCD deaths (28 million) occur (World Health Organization, 2013). While metabolic/physiological risk factors (e.g. high blood pressure) increase the risks of NCDs, modifiable behavioral risk factors such as tobacco use, physical inactivity, the harmful use of alcohol and unhealthy diet all increase the risk of dying from NCDs. Tobacco smoking alone causes around six million deaths per year (including deaths due to exposure to secondhand smoke), and is projected to kill eight million people a year by 2030 (World Health Organization, 2015). It is now the leading cause of cancer mortality and other chronic diseases in males worldwide (Islami et al., 2015; Takakura, 2015). Smoking is one of the main risk factor for lung cancer, stroke, heart disease, chronic respiratory disease, and diabetes (Al-Zalabani et al., 2015).

Overall, although different countries are at different stages of their tobacco epidemic, smoking prevalence is increasing across the world, with an estimate smoking prevalence of $51.1 \%$ among men and $7.9 \%$ among women aged 15 years and over as of 2015 (World Health Organization, 2015). Low- and middle-income countries are mostly in the earlier stages, with smoking prevalence increasing, therefore the proportion of tobacco-attributable deaths in those countries is expected to increase (Ayers et al., 2012). Cigarette smoking is reported to be the numberone risk factor that is attributed to more than four of five cases of lung cancer (Mendez et al., 2013; Xie et al., 2014).

Men are more likely to smoke cigarette than women in all communities, most possibly because of their working conditions or emotional status, and are thus

${ }^{1}$ Prevention of Psychosocial Injuries Research Center, Ilam University of Medical Sciences, Ilam, Iran ${ }^{2}$ Rehabilitation Counselling Discipline, Division of Health Systems and Global Populations, Faculty of Health Sciences, University of Sydney, Sydney, Australia ${ }^{3}$ Research Center for Health Sciences, Department of Epidemiology, School of Health, Shiraz University of Medical Sciences, Shiraz, ${ }^{4}$ Department of Epidemiology, School of Health change to Public health, Iran University of Medical Sciences, Tehran, Iran *For correspondence: Waliehmenati@yahoo.com 
more susceptible to smoke-related illnesses (Al-Naggar et al., 2014; Zhang et al., 2015). However, there is a considerable discrepancy in the prevalence of cigarette smoking in men across the world ( $\mathrm{Ng}$ et al., 2014). Although many developed and high-income countries, have reported a substantial decrease in the prevalence of cigarette smoking among the males, the rate is notably high I low- and middle-income countries (Islami et al., 2015). For example, in the US context, men are heavier cigarette smokers (18.8\%) than women (14.8\%) in 2014 (Agaku et al., 2014). In South Africa, however, cigarette smoking is reported to be $31.1 \%$ in men and $6.4 \%$ in women (World Health Organization, 2015).

The prevalence of cigarette smoking also differs in Iranian men, and ranges from $6.3 \%$ to $38.5 \%$ in recent years. A recent meta-analysis study of Iranian population shows that the cigarette smoking prevalence was about $19.8 \%$ in men (Moosazadeh et al., 2013). Similar srudy shows that thecigarrete smoking prevalence was $25.70 \%$ (CI95\%:23.74-27.66) among men aged 40-64 years old in Shahroud, Iran (Hamrah et al., 2013).

The prevalence of cigarette smoking is even different across and within countries by age, education level, ethnicity and race, socio-economic status, sexual orientation and so on (Agaku et al., 2014; Garrett et al., 2013). Yet, our understanding of the possible factors associated with smoking behavior remains shallow but it continues to be of critical importance to authorities towards developing preventive interventions in health care (Islami et al., 2015).

Previous literature has broadly recognized the role of age, gender, socio-economic status (i.e. income, education and employment), culture, demographic factors, and social determinants of health in male health-related behaviors such as cigarette smoking (McPherson et al., 2013; Lindstrom et al., 2014; Alvur et al., 2014). While some of these factors directly contribute to smoking behavior, the remaining may only act as a mediate or confounder factors towards the prevalence of smoking (Honjo et al., 2006). For example, Giordano and Lindstrom (2010) found that the prevalence of cigarette smoking was significantly higher among unemployed men than employed counterparts. Exiting literature, however, shows variations in the strength and direction of the associations between marriage and cigarette smoking behavior. Although some studies indicate that getting married can reduce the prevalence of cigarette smoking in men, others show no reduction in cigarette smoking with marriage (Giordano and Lindstrom, 2010; Pennanen et al., 2014).

The prevalence of overweight and/or obese is also increasing in nearly all countries (Jaacks et al., 2015). In Iran, it is estimated that $10.7 \%$ (CI 95\%: $8.8-12.5$ ) of men or people are obese (Rahmani et al., 2015). Researchers have identified many relevant factors that contribute to energy imbalance and weight gain. Examples include genetics, eating habits, place of living, attitudes and emotions, lifestyle and socio-economic status (Panel, 1998). The evidence also suggest associations between smoking and lower body weight, and smoking cessation and weight gain but those studies are predominately based on data collected in Western communities (Xu et al., 2007).
It is believed that smoking increases an individual's metabolic rate but it reduces the consumption of food materials. The cigarette smoking is therefore presumed to correlate with lower BMI. Although, there is some evidence to support the negative association between smoking and weight-related outcomes (Schindler-Ruwisch et al., 2015), some health researchers have not confirmed any associations between obesity and smoking (Li et al., 2010). Often, the associations between cigarette smoking and obesity relies on data obtained from Body Mass Index (BMI) calculations; however, the likely trends of obesity have not yet been well-quantified using measured BMI. To our knowledge, limited investigation has been conducted using representative data collected in developing countries.

This study aims to determine the prevalence of cigarette smoking and its relationship with individuals' demographic factors and BMI in the adolescent men living in Tehran, Iran.

\section{Materials and Methods}

\section{Data collection}

This study involved the secondary analysis of the 'Urban Health Equity Assessment and Response Tool2' (Urban HEART-2) survey conducted in Tehran, the capital city of Iran, among men aged 20+, 2011-2012. The pooled data were used to extract data about obesity and cigarette smoking behavior in adolescent males. The base cross-sectional study (2011) was initially conducted to explore the differences in health outcome and well-being in male population. The study sample included 45,590 men living in 22 districts of Tehran. Using a multistage sampling method, we first applied stratified sampling and considered each of the 22 districts as a strata, after which two hundred blocks were selected randomly from each district through the cluster sampling method. Subsequent sampling strategy included random selection of eight households from each block. All men in each family were then asked to complete the questionnaire (Asadi-Lari et al., 2013). This sample size was considered adequate to produce representative estimates for each district.

\section{Measures}

Cigarette-smoking behavior was measured based on two main questions: a) "have you smoked at least 100 cigarettes in your entire life?" and b) "do you smoke cigarettes every day, some days, or not at all?. We used the 'yes' and 'no'responses to these items to distinguish smokers from nonsmokers. Those who smoked at least five cigarettes per week were considered as cigarette smokers, and the remainder was considered as nonsmokers.

Several independent variables including age, marital status, education, employment status, region, place and time of residency in the district, height, weight, house ownership, nationality and family size were used to determine factors that contributed to smoking behavior. Data on height and weight were collected to measure BMI. The researcher used self-reported questions to obtain information concerning height and weight including a) how tall are you without shoes?" and b) "how much do 
you weigh without shoes?".

The World Health Organization BMI classification system for Asian countries was applied to identify adult underweight, overweight and obesity (WHO Expert Consultation, 2004): That is, underweight refers to a person whose BMI is below $18.5 \mathrm{~kg} / \mathrm{m}^{2}$; normal weight ranges from 18 to $23 \mathrm{~kg} / \mathrm{m}^{2}$; overweight ranges from 23 to $25 \mathrm{~kg} / \mathrm{m}^{2}$; and obese refers to a person whose BMI is above $25 \mathrm{~kg} / \mathrm{m}^{2}$ (Jang et al., 2012).

Ethics approval was granted from the Ethics Committee at the Shiraz University of Medical Sciences, Iran prior to the commencement of the data collection. Informed consent was obtained from all participants.

\section{Data analysis}

Data were imported to SPSS software version 21 for statistical analysis. Descriptive statistics, chi-square, t-test, and logistic regression model were computed. Logistic regression was used to examine the relationships between the independents variables and cigarette smoking behavior. Multivariate analysis was also employed to determine whether a potential confounding variable is associated with the exposure to cigarette smoking. Kolmogorov-Smirnov statistical test was used to confirm the normality of the independent, quantitative variables. Odds ratio with $95 \%$ confidence interval (CI 95\%) was considered for the interpretation of findings. Significance level was set at $\mathrm{p}<0.05$.

\section{Results}

A total of 45,990 men were included in the study. Mean age, BMI, and family size were $42.72 \pm 3.99$ years, $25.39 \pm 4.08$, and $3.99 \pm 1.37$ persons, respectively. The majority and minority of the participants were respectively living in district $18(\mathrm{~N}=2403 ; 5.24 \%)$ and district 22 $(\mathrm{N}=1666 ; 3.63 \%)$. In terms of ethnicity, almost $55.36 \%$ of the men were Fars and $25.38 \%$ were Azeri. We found

Table 1. Characteristics of the Smoker and Nonsmoker men in Tehran city.

\begin{tabular}{|c|c|c|c|c|c|c|}
\hline Characteristics & Group & Smoker n $(\%)$ & Nonsmoker n $(\%)$ & $\mathrm{P}$-value & OR crude & CI $95 \%$ \\
\hline \multirow{2}{*}{ Nationality } & Iranian & $6609(14.62)$ & $38610(85.38)$ & 0.98 & 0.99 & $0.80-1.24$ \\
\hline & Non-Iranian & $94(16.64)$ & $548(85.36)$ & & & \\
\hline \multirow{2}{*}{ House Ownership } & No & $2640(18.79)$ & $11413(81.21)$ & 0.01 & 1.58 & $1.49-1.66$ \\
\hline & Yes & $4075(12.80)$ & $27756(8720)$ & & & \\
\hline \multirow{5}{*}{ Age } & $20-30$ & $891(6.21)$ & 13456(93.79) & Base & - & - \\
\hline & $30-40$ & $1398(15.71)$ & $7503(84.29)$ & 0.01 & 0.35 & $0.32-0.39$ \\
\hline & $40-50$ & $1838(22.35)$ & $6387(77.65)$ & 0.01 & 0.23 & $0.21-0.25$ \\
\hline & $50-60$ & $1547(22.05)$ & $5468(77.95)$ & 0.01 & 0.23 & $0.21-0.26$ \\
\hline & $>60$ & $1049(13.98)$ & $6453(86.02)$ & 0.01 & 0.41 & $0.37-0.45$ \\
\hline \multirow{5}{*}{ Education status } & Illiterate & $413(17.35)$ & $1967(82.65)$ & Base & - & - \\
\hline & Primary school & $715(18.64)$ & $3120(81.36)$ & 0.19 & 0.91 & $0.80-1.05$ \\
\hline & Middle school & $1187(21.08)$ & $4444(78.92)$ & 0.01 & 0.41 & 0.37 .0 .44 \\
\hline & High school and Diploma & $3160(16.14)$ & $16417(83.86)$ & 0.13 & 1.09 & $0.98-1.22$ \\
\hline & Academic & $1217(8.51)$ & 13090(91.49) & 0.01 & 2.59 & $2.01-2.55$ \\
\hline \multirow{3}{*}{ Marital Status } & Married & $5526(18.34)$ & $24610(81.66)$ & Base & - & - \\
\hline & Single & $924(6.66)$ & 12949(93.34) & 0.01 & 3.14 & $2.92-3.38$ \\
\hline & Widow and divorced & $228(20.07)$ & $908(79.93)$ & 0.04 & 0.89 & $0.73-0.98$ \\
\hline \multirow{5}{*}{ Job status } & Unemployed & 791(14.71) & $4587(85.29)$ & Base & - & - \\
\hline & Student & $70(2)$ & $3415(98)$ & 0.01 & 8.41 & $6.56-10.78$ \\
\hline & Retirement & $1309(15.90)$ & $6923(84.10)$ & 0.06 & 0.91 & 0.82 .1 .01 \\
\hline & Employment & $3423(12.41)$ & $241540(87.59)$ & 0.02 & 1.22 & $1.13-1.32$ \\
\hline & Other & $103(11.88)$ & $764(88.12)$ & 0.03 & 1.28 & $1.03-1.59$ \\
\hline \multirow{4}{*}{ BMI } & Underweight & $144(15.48)$ & $786(84.71)$ & 0.88 & 0.98 & $0.81-1.20$ \\
\hline & Normal & $2196(10.34)$ & 19046(89.66) & 0.02 & 1.57 & $1.39-1.78$ \\
\hline & Overweight & $2390(14.21)$ & $14427(85.79)$ & 0.06 & 1.09 & $0.99-1.19$ \\
\hline & Obese & 794(15.29) & $4150(84.71)$ & Base & - & - \\
\hline
\end{tabular}

Base $=$ Reference group, $\mathrm{OR}=$ Odds Ratio; $\mathrm{CI}=$ Confidence Interval

Table 2. The Relationships Between Study Quantitative Variables and Cigarette Smoking Status in the Men of Tehran City

\begin{tabular}{|l|c|c|c|c|c|c|}
\hline \multirow{2}{*}{ Factors } & \multicolumn{2}{|c|}{ Smokers } & \multicolumn{2}{c|}{ Nonsmokers } & \multirow{2}{*}{ P-value } \\
\cline { 2 - 6 } & Mean & SD & Mean & 16.86 & 26.15 \\
\hline Age (year) & 46.86 & 13.51 & 42.01 & 1.38 & -4.96 & 0.01 \\
\hline Family size & 3.91 & 1.28 & 4 & 4.05 & 6.22 & 0.10 \\
\hline BMI & 25.32 & 4.21 & 25.41 & 1.25 & 2.12 \\
\hline Residency years & 3.94 & 1.23 & 4.06 & & 0.22 \\
\hline
\end{tabular}


Table 3. Analysis of the Exploratory Factors and the Cigarette Smoking Status in the ?en of Tehran Using Logistic Regression

\begin{tabular}{|c|c|c|c|c|c|c|}
\hline Variables & Subgroup & $\mathrm{B}^{*}$ & $\mathrm{SE}^{* *}$ & P-value & OR adjusted & CI \%95 \\
\hline Constant & - & 2.68 & 0.16 & 0.01 & 14.59 & - \\
\hline Family size & - & -0.15 & 0.01 & 0.15 & 0.98 & $0.96-1.01$ \\
\hline Age (per year) & - & -0.01 & 0.01 & 0.01 & 0.96 & $0.94-0.98$ \\
\hline BMI & - & 0.02 & 0.01 & 0.11 & 1.04 & $0.97-1.05$ \\
\hline House ownership & Yes/no & -0.37 & 0.03 & 0.01 & 0.68 & $0.64-0.72$ \\
\hline District & - & 0.01 & 0.01 & 0.76 & 1.01 & $0.99-1.02$ \\
\hline \multirow{4}{*}{ Job Status } & Employment / unemployed & -0.51 & 0.12 & 0.01 & 0.60 & $0.46-0.76$ \\
\hline & Student/ unemployed & -0.20 & 0.11 & 0.02 & 0.81 & $0.76-0.89$ \\
\hline & Retirement/ unemployed & 0.93 & 0.17 & 0.01 & 2.54 & $1.81-3.54$ \\
\hline & Other/ unemployed & 0.06 & 0.11 & 0.59 & 1.06 & $0.84-1.64$ \\
\hline \multirow{4}{*}{ Education level } & Primary school & -0.34 & 0.07 & 0.01 & 0.71 & $0.62-0.81$ \\
\hline & Middle school & -0.48 & 0.06 & 0.01 & 0.62 & $0.55-0.69$ \\
\hline & High school and diploma & -0.70 & 0.05 & 0.01 & 0.50 & $0.45-0.54$ \\
\hline & Academic & -0.53 & 0.04 & 0.01 & 0.59 & $0.54-0.63$ \\
\hline \multirow{2}{*}{ Marital Status } & Married /widow and divorced & -0.85 & 0.05 & 0.01 & 0.42 & $0.39-0.47$ \\
\hline & Married/ single & -0.92 & 0.09 & 0.01 & 0.40 & $0.33-0.48$ \\
\hline
\end{tabular}

*Unstandardized coefficient, **Standard Error

that the overall prevalence of cigarette smoking was about 14.62 (CI95\%: 14.29-14.94) in the men ( $n=6,723)$. The overall prevalence of obesity (BMI>25) was 11.16 $\%(n=4,899)$. Other demographic characteristics and the association between exploratory factors and cigarette smoking behavior has been shown in Table 1 .

Table 1 indicates the findings from bivariate analysis in that house ownership status, age group, marital status, education level, and job status associate with the prevalence of cigarette smoking. However, the associations between BMI, nationality and cigarette smoking behavior is not statistically significant. The students had the highest risk of exposure to cigarette smoking compared to others groups (OR=8.41).

The characteristics of the quantitative variables and their relationships with cigarette smoking behavior are presented in Table 2 .

The mean age of the smoker group was higher than that of the nonsmoker group (46.86vs. 42.01 years). There was a significant association between age and cigarette smoking status $(\mathrm{p}=0.01)$. The average of family size had a statistically significant association with cigarette smoking in the men $(\mathrm{p}=0.01)$. The mean residency $(\mathrm{p}=0.22)$ and BMI $(p=0.10)$, however, were not different in the smoker and nonsmoker groups.

The significant variables $(\mathrm{p} \leq 0.05)$ and those close to significant variables $(\mathrm{p} \leq 0.2)$ from the univariate analysis stage were then included into logistic regression model in order to assess the simultaneous relationships among variables. Table 3 depicts the findings of the multivariate analysis through logistic regression. Collinearity statistics were checked to determine the existence of multicollinearity (if two or more independents variables in a regression model are highly correlated, is accrued collinearity). No collineriaty was found among the independents variables since most of the correlations were $<0.30$.

Findings of the logistic regression indicate that BMI was significantly associated with cigarette smoking behavior $(\mathrm{OR}=1.04)$. Also, there were also statistical relationships between adolescents' cigarette smoking behavior and their age, house ownership, job status, marital status and qualifications. However, no significant associations was found between family size or district and the smoking status.

\section{Discussion}

This study aimed to examine the prevalence of cigarette smoking in male adolescents living in Tehran, and explore the associations between participants' demographic factors, BMI and cigarette-smoking behavior. Our findings added to a growing body of literature that highlights the association between demographic factors and cigarette smoking in men. In the present study, the prevalence of cigarette smoking was 14.62 (CI95\%: 14.29-14.94) in the men $(n=6,723)$. This rate is slightly lower than those found in previous research. In a meta-analysis conducted among Iranian population, Moosazadeh and collaguses (2013) estimated the prevalence of cigarette smoking to be around $19.8 \%$ in the men. In China, Jiang (2015) found that the cigarette smoking prevalence is $22.57 \%$ among male (Jiang et al., 2015). Likewise, a Sweden-based study indicated the smoking prevalence to be $14.9 \%$ among men (Lindstrom, 2010).

Given the cigarette smoking prevalence has slightly declined amongst men living in Tehran, it is still a high rate which remains a significant public health concern. 
Our findings indicated that demographic characteristics are significantly associated with cigarette smoking behavior in men, as found by previous studies (Jalilian et al., 2015: Mohsin and Bauman, 2005).

The bivariate analysis in the present study indicated that demographic variables including age, family size and BMI were related to cigarette smoking status in the men, with higher Means in the smokers than in the nonsmokers. We found that the cigarette smoking prevalence was $21.08 \%(n=1187)$ among men with middle school education and $18.64 \%(n=715)$ among those with primary school education, reflecting the direct relationship between education level and smoking status. The prevalence of smoking in the divorced men was higher than those who were married or single, indicating the relationship between marital statuses and smoking behavior. Jalilian also indicated that educational level was the most significant predicators for cigarette smoking among male students in Iran; the male students with higher education level had less probability of smoking (Jalilian et al., 2015).

We also found different associations between the study demographic factors and cigarette smoking behavior. According to the logistic regression model, age, BMI, house ownership, job 1 (employment/unemployed), job 2(student/unemployed), job 3 (retirement/unemployed), education 1 (primary school /illiterate), Education 2 (mid school/illiterate), education 3 (high school and diploma / illiterate), education 4 (academic/illiterate), marital status 1 (married /widow and divorced) and marital status 2 (married/single) were associated with cigarette-smoking behavior in the men, even though variables family size and district were not statistically associated with smoking in the multivariate analysis. These findings are in line with those of previous studies. Lindstrom (2010), for example, found that daily cigarette smoking was significantly more in middle-aged men than in younger men (Lindstrom, 2010).

Richter and Leppin (2007) also found that education was a significant predictor of cigarette smoking among German male students. Never-married and divorced men had also a significantly higher prevalence of daily cigarette smoking than married men. Low socioeconomic status presumed to be a significant mediator on the association between marital status and daily cigarette smoking (Richter and Leppin, 2007).

According to our findings, the Mean differences in BMI between smokers and nonsmokers was not statistically significant (OR=1.04, CI95\%:0.97-1.05). Similar studies have found the same results. SchindlerRuwisch et al. (2015), for instance, reported that the Mean BMI for never, intermittent, and daily cigarette smokers were 26.3,27.4 and 28.1, respectively, reflecting that cigarette smokers had BMIs similar to the general population (Schindler-Ruwisch et al., 2015). Li et al.'s study (2010) on Mongolian population in China also did not provide any evidence to support an association between cigarette smoking and obesity. Only few studies have found a negative and/or positive association between BMI and cigarette smoking (Sohn, 2015), reflecting that future studies could look into the exact mechanisms that underlie the association between obesity and cigarette smoking.

In this study, the difference observed between the findings obtained from the bivariate analysis and those of logistic regression (OR crude and OR adjusted) is most likely because of the role of other confounders. The major strength of the present study however is its use of national representativeness of the database in Iran. There were several limitations associated with this study: a) the prevalence of obesity and cigarette smoking behavior was measured based on self-report data which introduce bias; and b) the study applied a cross-sectional design, in which both independent variables and cigarette smoking status were measured simultaneously, meaning that the issue of temporality was not considered in the examination of relationships.

Given the relatively high prevalence of cigarette smoking in the study population, policy interventions are required to address this major public health issue, with a focus on the population demographic influences. Effective tobacco control policies must be implemented or enforced, especially amongst young people in order to reduce the prevalence of cigarette smoking and associated risks. Further research is suggested to identify the protective mechanisms that influence on the association between demographic factors and cigarette smoking status. Both widowed and divorced men seem to be an important target population for smoking prevention. How specific demographic factors may be linked to cigarette smoking, and which mechanisms increase the effectiveness of smoking prevention, are suggested for future studies.

\section{Acknowledgements}

This article approved by the Research Vice-Chancellor of Shiraz University of Medical Sciences (Proposal No. 32-968).

\section{References}

Agaku IT, King BA, Dube SR, et al (2014). Current cigarette smoking among adults-United States, 2005-2012. MMWR Morb Mortal Wkly Rep, 63, 29-34.

Al-Naggar RA, Bobryshev YV, Anil S (2014). Pattern of shisha and cigarette smoking in the general population in Malaysia. Asian Pac J Cancer Prev, 15, 10841-6.

Al-Zalabani AH, Abdallah AR, Alqabshawi RI (2015). Intention to Quit Smoking among Intermediate and Secondary School Students in Saudi Arabia. Asian Pac J Cancer Prev, 16, 6741-7.

Alvur TM, Cinar N, Oncel S, et al (2014). Trends in smoking among university students between 2005-2012 in Sakarya, Turkey. Asian Pac J Cancer Prev, 15, 4575-81.

Asadi-Lari M, Vaez-Mahdavi MR, Faghihzadeh S, et al (2013). Response-oriented measuring inequalities in Tehran: second round of Urban Health Equity Assessment and Response Tool (Urban HEART-2), concepts and framework. Med $J$ Islamic Republic Iran, 27, 236-48.

Ayers JW, Althouse BM, Allem JP, et al (2012). A novel evaluation of World No Tobacco day in Latin America. $J$ Med Internet Res, 14, 42-66.

Chen CY, Wu CC, Chang HY, et al (2014). The effects of social structure and social capital on changes in smoking status from 8th to 9th grade: Results of the Child and Adolescent 
Aziz Kassani et al

Behaviors in Long-term Evolution (CABLE) study. Prev Med, 62, 148-54.

Garrett BE, Dube SR, Winder C, et al (2013). Cigarette SmokingUnited States, 2006-2008 and 2009-2010. CDC Health Disparities Inequalities Report-United States, 62, 81.

Giordano GN, Lindstrom M (2010). The impact of social capital on changes in smoking behaviour: a longitudinal cohort study. Eur J Public Health, 48.

Hamrah MS, Harun-Or-Rashid M, Hirosawa T, et al (2013). Smoking and associated factors among the population aged 40-64 in shahroud, Iran. Asian Pac J Cancer Prev, 14, 1919-23.

Honjo K, Tsutsumi A, Kawachi I, et al (2006). What accounts for the relationship between social class and smoking cessation? Results of a path analysis. Social Science Med, 62, 317-28.

Islami F, Torre LA, Jemal A (2015). Global trends of lung cancer mortality and smoking prevalence. Translational Lung Cancer Res, 4, 327.

Jaacks LM, Slining MM, Popkin BM (2015). Recent underweight and overweight trends by rural-urban residence among women in low-and middle-income countries. J Nutrit, 145, 352-7.

Jalilian F, Karami Matin B, Ahmadpanah M, et al (2015). Socio-demographic characteristics associated with cigarettes smoking, drug abuse and alcohol drinking among male medical university students in Iran. J Res Health Sci, 15, 42-6.

Jang SY, Kim JH, Lim MK, et al (2012). Relationship between BMI, body image, and smoking in Korean women as determined by urine cotinine: results of a nationwide survey. Asian Pac J Cancer Prev, 13, 1003-10.

Jiang G, Wang D, Xu Z, et al (2015). Work environments and mortality attributable to smoking: a study among male citizens from 2010 to 2012 in Tianjin, China. J Industrial Hygiene Occupational Diseases, 33, 241-4 [in Chinese].

Li H, Tong W, Wang A, et al (2010). Effects of cigarette smoking on blood pressure stratified by BMI in Mongolian population, China. Blood Pressure, 19, 92-7.

Lindstrom M (2010). Social capital, economic conditions, marital status and daily smoking: a population-based study. Public Health, 124, 71-7.

Lindstrom M, Axelsson J, Moden B, et al (2014). Sexual orientation, social capital and daily tobacco smoking: a population-based study. BMC Public Health, 14, 565.

McPherson KE, Kerr S, Morgan A, et al (2013). The association between family and community social capital and health risk behaviours in young people: an integrative review. $B M C$ public health, 13, 971.

Mendez D, Alshanqeety O, Warner KE (2013). The potential impact of smoking control policies on future global smoking trends. Tobacco Control, 22, 46-51.

Mohsin M, Bauman AE (2005). Socio-demographic factors associated with smoking and smoking cessation among 426,344 pregnant women in New South Wales, Australia. BMC Public Health, 5, 138.

Moosazadeh M,Ziaaddini H, Mirzazadeh A, et al (2013). Metaanalysis of smoking prevalence in Iran. Addict Health, $\mathbf{5}$, 140.

$\mathrm{Ng} \mathrm{M}$, Freeman MK, Fleming TD, et al (2014). Smoking prevalence and cigarette consumption in 187 countries, 1980-2012. Jama, 311, 183-92.

Panel NOEIE (1998). Clinical guidelines on the identification, evaluation, and treatment of overweight and obesity in adults.

Pennanen M, Broms U, Korhonen T, et al (2014). Smoking, nicotine dependence and nicotine intake by socio-economic status and marital status. Addict Behav, 39, 1145-51.
Richter M, Leppin A (2007). Trends in socio-economic differences in tobacco smoking among German schoolchildren, 19942002. Eur J Public Health, 17, 565-71.

Schindler-Ruwisch J, Augustson E, Lynch K, et al (2015). BMI and Smoking: Interrelated Factors among Cessation Website Users. Am J Health Behavior, 39, 330-7.

Sohn K (2015). The effects of smoking on obesity: evidence from Indonesian panel data. Tobacco Induced Diseases, 13, 1-8.

Takakura M (2015). Relations of participation in organized activities to smoking and drinking among Japanese youth: contextual effects of structural social capital in high school. Int J Public Health, 60, 679-89.

Thun M, Peto R, Boreham J, et al (2012). Stages of the cigarette epidemic on entering its second century. Tobacco Control, 21, 96-101.

WHO Expert Consultation (2004). Appropriate body-mass index for Asian populations and its implications for policy and intervention strategies. Lancet, 363, 157-63.

World Health Organization (2013). Global action plan for the prevention and control of noncommunicable diseases 2013-2020.

World Health Organization (2015). WHO global report on trends in prevalence of tobacco smoking 2015.

Xie D, Lan L, Huang K, et al (2014). Association of p53/p21 expression and cigarette smoking with tumor progression and poor prognosis in non-small cell lung cancer patients. Oncol Reports, 32, 2517-26.

Xu F, Xiao-Mei Y, Wang Y (2007). The association between amount of cigarettes smoked and overweight, central obesity among Chinese adults in Nanjing, China. Asia Pac J Clin Nutrit, 16, 240.

Zhang F, Zhang T, Xiong J, et al (2015). Analysis on prevalence and influence factors of smoking and drinking among middle shool students of Tujia and Miao in Xiangxi Area. Wei sheng yan jiu. J Hygiene Res, 44, 750-66 [in Chinese]. 